\title{
QUANTUM MARKOVIAN SEMIGROUPS ON QUANTUM SPIN SYSTEMS: GLAUBER DYNAMICS
}

\author{
Veni Choi, Chul Ki Ko, and Yong Moon Park
}

\begin{abstract}
We study a class of KMS-symmetric quantum Markovian semigroups on a quantum spin $\operatorname{system}(\mathcal{A}, \tau, \omega)$, where $\mathcal{A}$ is a quasi-local algebra, $\tau$ is a strongly continuous one parameter group of *-automorphisms of $\mathcal{A}$ and $\omega$ is a Gibbs state on $\mathcal{A}$. The semigroups can be considered as the extension of semigroups on the nontrivial abelian subalgebra. Let $\mathcal{H}$ be a Hilbert space corresponding to the GNS representation constructed from $\omega$. Using the general construction method of Dirichlet form developed in [8], we construct the symmetric Markovian semigroup $\left\{T_{t}\right\}_{t \geq 0}$ on $\mathcal{H}$. The semigroup $\left\{T_{t}\right\}_{t \geq 0}$ acts separately on two subspaces $\mathcal{H}_{d}$ and $\mathcal{H}_{\text {od }}$ of $\mathcal{H}$, where $\mathcal{H}_{d}$ is the diagonal subspace and $\mathcal{H}_{\text {od }}$ is the off-diagonal subspace, $\mathcal{H}=\mathcal{H}_{d} \oplus \mathcal{H}_{\text {od }}$. The restriction of the semigroup $\left\{T_{t}\right\}_{t \geq 0}$ on $\mathcal{H}_{d}$ is Glauber dynamics, and for any $\eta \in \mathcal{H}_{o d}, T_{t} \eta$ decays to zero exponentially fast as $t$ approaches to the infinity.
\end{abstract}

\section{Introduction}

A KMS symmetric quantum Markovian semigroup $\left\{S_{t}\right\}_{t \geq 0}$ on a von Neumann algebra $\mathcal{M}$ is a KMS symmetric, weakly continuous, contractive and identity preserving semigroup on $\mathcal{M}$ [6]. Quantum Markovian semigroups are the natural generalization of classical Markovian semigroups and were introduced in physics to model the decay equilibrium of quantum open systems $[2,3,6,8,9]$.

Many mathematicians and physicists are interested to the problems whether quantum Markovian semigroups on the subalgebra of a von Neumann algebra or a $C^{*}$-algebra have their extensions on the full algebra. The problem of the extension was studied in $[1,5,7]$. In [5], authors constructed a special class of generic quantum Markovian semigroups arising in the stochastic limit of a discrete system with generic free Hamiltonian interacting with a mean zero, gauge invariant, Gaussian field, and studied its properties. The semigroups are constructed on the algebra $B(h)$ of all bounded operators on a complex

Received December 1, 2006.

2000 Mathematics Subject Classification. 46L55, 37A60.

Key words and phrases. KMS symmetric quantum Markovian semigroups, quantum spin systems, diagonal subspace, Glauber dynamics. 
separable Hilbert space $h$ and leave invariant not only the diagonal subalgebra but also the off diagonal subspace with respect to a fixed basis of $h$. The action on diagonal operators describes a classical Markov jump process. Goderis and Maes [7] studied a quantum dynamical system which is an extension of the classical system such that the property of local reversibility is preserved.

Let $\mathcal{M}$ be a von Neumann algebra acting on a complex Hilbert space $\mathcal{H}$ and $\xi_{0}$ be a fixed cyclic and separating vector for $\mathcal{M}$. Let $\Delta$ and $J$ be the modular operator and the modular conjugation associated with the pair $\left(\mathcal{M}, \xi_{0}\right)$ [4]. Consider the symmetric embedding:

$$
i_{0}: \mathcal{M} \longrightarrow \mathcal{H}, \quad i_{0}(A)=\Delta^{1 / 4} A \xi_{0} .
$$

For a given KMS symmetric quantum Markovian semigroup $\left\{S_{t}\right\}_{t \geq 0}$ on $\mathcal{M}$, the semigroup $\left\{T_{t}\right\}_{t \geq 0}$ on $\mathcal{H}$ defined by

$$
T_{t} \circ i_{0}=i_{0} \circ S_{t}
$$

is symmetric, strongly continuous, positive preserving, contractive and $T_{t} \xi_{0}=$ $\xi_{0}$ for all $t \geq 0$. The semigroup $\left\{T_{t}\right\}_{t \geq 0}$ is called a symmetric Markovian semigroup on $\mathcal{H}$. Conversely, for a given symmetric Markovian semigroup $\left\{T_{t}\right\}_{t \geq 0}$ on $\mathcal{H}$, the semigroup $\left\{S_{t}\right\}_{t \geq 0}$ on $\mathcal{M}$ defined by

$$
i_{0} \circ S_{t}=T_{t} \circ i_{0}
$$

is a KMS symmetric quantum Markovian semigroup. (See Theorem 2.11 and Theorem 2.12 of [6].)

The purpose of this paper is to study a class of KMS symmetric quantum Markovian semigroups on a quantum spin system $(\mathcal{A}, \tau, \omega)$, where $\mathcal{A}$ is a quasi-local algebra, $\tau$ is a strongly continuous one parameter group of $*-$ automorphisms of $\mathcal{A}$ and $\omega$ is a Gibbs state on $\mathcal{A}$. The semigroups can be considered as the extension of semigroups on the nontrivial abelian subalgebra. Let $\mathcal{H}$ be a Hilbert space corresponding to the GNS representation constructed from $\omega$. Using the general construction method of Dirichlet forms developed in [8] (noncommutative Dirichlet form in the sense of Cipriani [6]), we construct the symmetric Markovian semigroup $\left\{T_{t}\right\}_{t \geq 0}$ on $\mathcal{H}$. The semigroup $\left\{T_{t}\right\}_{t>0}$ acts separately on two subspaces $\mathcal{H}_{d}$ and $\mathcal{H}_{o d}$ of $\mathcal{H}$, where $\mathcal{H}_{d}$ is the diagonal subspace and $\mathcal{H}_{o d}$ is the off-diagonal subspace, $\mathcal{H}=\mathcal{H}_{d} \oplus \mathcal{H}_{o d}$. The restriction of the semigroup $\left\{T_{t}\right\}_{t \geq 0}$ on $\mathcal{H}_{d}$ is Glauber dynamics, and for any $\eta \in \mathcal{H}_{o d}$, $T_{t} \eta$ decays to zero exponentially fast as $t \rightarrow \infty$.

This paper is organized as follows. In Section 2, we introduce a quantum spin system, and construct the symmetric Markovian semigroups by employing the general construction method of Dirichlet forms developed in [8] on standard forms of von Neumann algebras. In Section 3, we give the concrete action of the semigroup constructed in Section 2 and investigate some properties of the semigroup. 


\section{Quantum Markovian semigroups}

In this section, we introduce a quantum spin system and construct the symmetric Markovian semigroup by employing the general construction method of Dirichlet forms developed in [8] on standard forms of von Neumann algebras.

Let $\mathbb{M}_{2}(\mathbb{C})$ be the algebra of $2 \times 2$ matrices with complex entries. Any $2 \times 2$ matrix is decomposed as a linear combination of the Pauli matrices $S^{0}, S^{x}, S^{y}, S^{z}$ defined by

$$
S^{0}=\mathbf{1}=\left(\begin{array}{ll}
1 & 0 \\
0 & 1
\end{array}\right), S^{x}=\left(\begin{array}{cc}
0 & 1 \\
1 & 0
\end{array}\right), S^{y}=\left(\begin{array}{cc}
0 & -i \\
i & 0
\end{array}\right), S^{z}=\left(\begin{array}{cc}
1 & 0 \\
0 & -1
\end{array}\right) .
$$

We define projection operators on $\mathbb{C}^{2}$ with an inner product $(\cdot, \cdot)$ :

$$
\begin{gathered}
N=|u\rangle\left\langle u\left|=\left(\begin{array}{ll}
1 & 0 \\
0 & 0
\end{array}\right), \mathbf{1}-N=\right| d\right\rangle\langle d|=\left(\begin{array}{ll}
0 & 0 \\
0 & 1
\end{array}\right), \\
b=|d\rangle\left\langle u\left|=\left(\begin{array}{ll}
0 & 0 \\
1 & 0
\end{array}\right), b^{*}=\right| u\right\rangle\langle d|=\left(\begin{array}{ll}
0 & 1 \\
0 & 0
\end{array}\right),
\end{gathered}
$$

where $u=\left(\begin{array}{l}1 \\ 0\end{array}\right), d=\left(\begin{array}{l}0 \\ 1\end{array}\right)$ and $|\eta\rangle\langle\xi|$ denotes the one rank operator on $\mathbb{C}^{2}$ such that $|\eta\rangle\langle\xi| \zeta=(\xi, \zeta) \eta$.

Let $\mathcal{F}$ be the family of bounded sets in $\mathbb{Z}^{\nu}$ :

$$
\mathcal{F}=\left\{\Lambda \in \mathbb{Z}^{\nu}: \Lambda \text { is finite }\right\} .
$$

For each $\Lambda \in \mathcal{F}, \mathcal{A}_{\Lambda}$ is the local $C^{*}$-algebra given by

$$
\mathcal{A}_{\Lambda}=\bigotimes_{k \in \Lambda} \mathbb{M}_{k}
$$

where $\mathbb{M}_{k}$ is an identical copy of $\mathbb{M}_{2}(\mathbb{C})$. We denote $\mathcal{A}_{\{k\}}$ by $\mathcal{A}_{k}, k \in \mathbb{Z}^{\nu}$. For $\Lambda_{1}, \Lambda_{2} \in \mathcal{F}, \Lambda_{1} \cap \Lambda_{2}=\emptyset, \mathcal{A}_{\Lambda_{1}}$ is isomorphic to the $C^{*}$-subalgebra $\mathcal{A}_{\Lambda_{1}} \otimes \mathbf{1}_{\Lambda_{2}}$ of $\mathcal{A}_{\Lambda_{1} \cup \Lambda_{2}}$, where $\mathbf{1}_{\Lambda_{2}}$ denotes the identity operator of $\mathcal{A}_{\Lambda_{2}}$. We identify $\mathcal{A}_{\Lambda_{1}}$ and $\mathcal{A}_{\Lambda_{1}} \otimes \mathbf{1}_{\Lambda_{2}}$. Then $\mathcal{A}_{\Lambda_{1}} \subset \mathcal{A}_{\Lambda_{2}}$ whenever $\Lambda_{1} \subset \Lambda_{2}$, and $\left[A_{1}, A_{2}\right]=0, A_{1} \in$ $\mathcal{A}_{\Lambda_{1}}, A_{2} \in \mathcal{A}_{\Lambda_{2}}$ whenever $\Lambda_{1} \cap \Lambda_{2}=\emptyset$. Here $[A, B]$ means $A B-B A$.

The quasi local algebra $\mathcal{A}$ is defined as the uniform closure of $\mathcal{A}_{0}$ :

$$
\begin{aligned}
\mathcal{A}_{0} & =\bigcup_{\Lambda \in \mathcal{F}} \mathcal{A}_{\Lambda}, \\
\mathcal{A} & =\overline{\mathcal{A}_{0}} .
\end{aligned}
$$

For each $k \in \mathbb{Z}^{\nu}$, the elements $S_{k}^{x}, S_{k}^{y}, S_{k}^{z}, N_{k}, b_{k}^{*}, b_{k}$ in $\mathbb{M}_{k}$ are identical copies of $S^{x}, S^{y}, S^{z}, N, b^{*}, b$, respectively, and we write $A_{k} \otimes \mathbf{1}_{\{k\}} \in \mathcal{A}_{k}$ as $A_{k}$.

We consider the Ising Hamiltonian: for $\Lambda \in \mathcal{F}$,

$$
\begin{aligned}
H_{\Lambda} & =-\beta \sum_{\substack{\{k, l\} \subset \Lambda \\
|k-l|=1}} S_{k}^{z} S_{l}^{z} \\
& =-\frac{\beta}{2} \sum_{\substack { l \in \Lambda \\
\begin{subarray}{c}{k \in \Lambda \\
|k-l|=1{ l \in \Lambda \\
\begin{subarray} { c } { k \in \Lambda \\
| k - l | = 1 } }\end{subarray}} S_{k}^{z} S_{l}^{z},
\end{aligned}
$$


where $\beta>0$ is an inverse temperature. Clearly $H_{\Lambda}$ is a self-adjoint element of $\mathcal{A}_{\Lambda}$. The time evolution $\tau_{t}$ is given by the strongly convergent limit of a one parameter semigroups $\tau_{t}^{\Lambda}$ on $\mathcal{A}_{\Lambda}$ such that

$$
\lim _{\Lambda \rightarrow \mathbb{Z}^{\nu}}\left\|\tau_{t}(A)-\tau_{t}^{\Lambda}(A)\right\|, A \in \mathcal{A},
$$

where $\tau_{t}^{\Lambda}(A)=e^{-i t H_{\Lambda}} A e^{i t H_{\Lambda}}, A \in \mathcal{A}_{\Lambda}, t \in \mathbb{R}$. (See Theorem 6.2.4 and Theorem 6.2 .6 of $[4]$.)

For $\Lambda \in \mathcal{F}$, define the local Gibbs state $\omega_{\Lambda}$ on $\mathcal{A}_{\Lambda}$ associated with $H_{\Lambda}$ by

$$
\omega_{\Lambda}(A)=\operatorname{Tr}\left(\rho_{\Lambda} A\right), A \in \mathcal{A}_{\Lambda},
$$

where $\rho_{\Lambda}=e^{H_{\Lambda}} / \operatorname{Tr}\left(e^{H_{\Lambda}}\right) . \omega_{\Lambda}$ has an extension $\tilde{\omega}_{\Lambda}$ to a state on $\mathcal{A}$ by Proposition 2.3.24 of [4]. Moreover there exist nets of extensions $\tilde{\omega}_{\Lambda_{\alpha}}$ of $\omega_{\Lambda_{\alpha}}$ such that $\tilde{\omega}_{\Lambda_{\alpha}}$ converges weakly* to a state $\omega$ on $\mathcal{A}$ :

$$
\lim _{\Lambda_{\alpha} \rightarrow \mathbb{Z}^{\nu}} \tilde{\omega}_{\Lambda_{\alpha}}(A)=\omega(A)
$$

for all $A \in \mathcal{A}$. Hence $\omega$ is a thermodynamic limit point of the local Gibbs states in the sense that

$$
\omega(A)=\lim _{\alpha} \omega_{\Lambda_{\alpha}}(A)
$$

for all $A \in \mathcal{A}_{\Lambda}$ and all $\Lambda \in \mathcal{F}$. The thermodynamic limit $\omega$ is a $\tau$-KMS state on $\mathcal{A}$ (Proposition 6.2 .15 of [4]).

Let $\left(\mathcal{H}_{\omega}, \pi_{\omega}, \Omega_{\omega}\right)$ be the GNS-representation (or cyclic representation) of $(\mathcal{A}, \omega)$ [4]. Throughout this paper, we write that $\mathcal{H}=\mathcal{H}_{\omega}, \xi_{0}=\Omega_{\omega}, \mathcal{M}_{\Lambda}=$ $\pi_{\omega}\left(\mathcal{A}_{\Lambda}\right), \mathcal{M}=\pi_{\omega}(\mathcal{A})^{\prime \prime}$ and $\tau_{t}^{\omega}=\pi_{\omega} \circ \tau_{t}$. To simplify the notations, we will omit $\pi_{\omega}$ such that $S_{k}^{z}:=\pi_{\omega}\left(S_{k}^{z}\right)$, etc. Denote by $\langle\cdot, \cdot\rangle$ the inner product of $\mathcal{H}$. Let $\sigma_{t}, t \in \mathbb{R}$, be the modular automorphism with respect to $\omega$ and $\Delta$ and $J$ be the modular operator and modular conjugation associated to the pair $\left(\mathcal{M}, \xi_{0}\right)[4]$, respectively. By Theorem 5.3.10 of [4], $\sigma_{t}=\tau_{t}^{\omega}$ and $\sigma_{t}(A)=\Delta^{i t} A \Delta^{-i t}, A \in$ $\mathcal{M}$. Let $\mathcal{M}^{\prime}$ be the commutant of $\mathcal{M}$. The map $j: \mathcal{M} \rightarrow \mathcal{M}^{\prime}$ is the anti-linear *-isomorphism defined by $j(A)=J A J, A \in \mathcal{M}$.

To construct a generator of a symmetric Markovian semigroup on $\mathcal{H}$, we introduce an (normalized) admissible function [8].

Definition 2.1. An analytic function $f: D \rightarrow \mathbb{C}$ on a domain $D$ containing the strip $I_{1 / 4}=\{z|| \operatorname{Im} z \mid \leq 1 / 4\}$ is said to be admissible if the following properties hold:

(a) $f(t) \geq 0$ for all $t \in \mathbb{R}$,

(b) $f(t+i / 4)+f(t-i / 4) \geq 0$ for all $t \in \mathbb{R}$,

(c) there exist $M>0$ and $p>1$ such that the bound

$$
|f(t+i s)| \leq M(1+|t|)^{-p}
$$

holds uniformly in $s \in[-1 / 4,1 / 4]$.

Moreover, if $\int f(t) d t=1$ then it is called a normalized admissible function. 
The function

$$
g(t)=\frac{2}{\sqrt{2 \pi}} \int\left(e^{k / 4}+e^{-k / 4}\right)^{-1} e^{-\frac{1}{2} k^{2}} e^{-i k t} d k
$$

is admissible. (See Lemma 3.1 of [8].)

For a fixed normalized admissible function $f$, we define an operator $H$ on $\mathcal{H}$ by

$$
\begin{aligned}
D(H) & =\left\{\xi \in \mathcal{H}: \sum_{k \in \mathbb{Z}^{\nu}}\left\|H_{k} \xi\right\|^{2}<\infty\right\} \\
H \xi & =\sum_{k \in \mathbb{Z}^{\nu}} H_{k} \xi, \xi \in D(H)
\end{aligned}
$$

where

$$
\begin{aligned}
H_{k}= & H_{k, 1}+H_{k, 2}+H_{k, 3}, \\
H_{k, l}=\int[ & \left.\sigma_{t+i / 4}\left(x_{k, l}^{*}\right)-j\left(\sigma_{t+i / 4}\left(x_{k, l}\right)\right)\right] \\
& \times\left[\sigma_{t-i / 4}\left(x_{k, l}\right)-j\left(\sigma_{t-i / 4}\left(x_{k, l}^{*}\right)\right)\right] f(t) d t
\end{aligned}
$$

for $k \in \mathbb{Z}^{\nu}, l=1,2,3$ and $x_{k, 1}=b_{k}^{*}, x_{k, 2}=b_{k}, x_{k, 3}=N_{k}$.

Theorem 2.2. Let $H$ be an operator defined as in (2.5) and (2.6). Then it is a generator of a strongly continuous, symmetric Markovian semigroup $\left\{T_{t}\right\}_{t \geq 0}, T_{t}=e^{-t H}$ on $\mathcal{H}$.

Proof. By Theorem 2.1 of [3], for each $k \in \mathbb{Z}^{\nu}, H_{k}$ is a (bounded) generator of a strongly continuous, symmetric Markovian semigroup on $\mathcal{H}$. See also Theorem 3.1 of [8]. Clearly $\mathcal{M}_{\Lambda} \xi_{0} \subset D(H)$ for any $\Lambda \in \mathcal{F}$, and so $H$ is densely defined. Since $H$ is a symmetric operator on $\mathcal{H}$ it has a closed extension, denoted by $H$ again. By Theorem 5.2 of [6], $H$ is a generator of a strongly continuous, symmetric Markovian semigroup $\left\{T_{t}\right\}_{t \geq 0}, T_{t}=e^{-t H}$ on $\mathcal{H}$.

\section{Action of the generator $H$}

In this section, we introduce two subspaces of $\mathcal{H}$, the diagonal subspace $\mathcal{H}_{d}$ and the off-diagonal subspace $\mathcal{H}_{o d}, \mathcal{H}=\mathcal{H}_{d} \oplus \mathcal{H}_{o d}$, and investigate the concrete action of the generator $H$ of the semigroup $\left\{T_{t}\right\}_{t \geq 0}$ constructed in Theorem 2.2 on $\mathcal{H}_{d}$ and $\mathcal{H}_{o d}$, respectively.

We first give elementary facts.

Lemma 3.1. (a) The following relations hold: for any $k \in \mathbb{Z}^{\nu}$

$$
\begin{aligned}
& \left(S_{k}^{z}\right)^{2}=1, b_{k}^{2}=\left(b_{k}^{*}\right)^{2}=0, \\
& b_{k} b_{k}^{*}=(1-N)_{k}=-S_{k}^{z}(1-N)_{k}, b_{k}^{*} b_{k}=N_{k}=S_{k}^{z} N_{k}, \\
& b_{k}^{*} S_{k}^{z}=-S_{k}^{z} b_{k}^{*}=-b_{k}^{*}, \quad b_{k} S_{k}^{z}=-S_{k}^{z} b_{k}=b_{k}, \\
& {\left[S_{k}^{z}, b_{k}^{*}\right]=2 b_{k}^{*},\left[S_{k}^{z}, b_{k}\right]=-2 b_{k} .}
\end{aligned}
$$


(b) The actions of modular operator $\sigma_{t}$

$$
\begin{aligned}
\sigma_{t}\left(b_{k}^{*}\right) & =\exp \left(i \beta t \sum_{\substack{l \in Z^{\nu} \\
|l-k|=1}} S_{l}^{z}\right) b_{k}^{*}, \\
\sigma_{t}\left(b_{k}\right) & =\exp \left(-i \beta t \sum_{\substack{l \in Z^{\nu} \\
|l-k|=1}} S_{l}^{z}\right) b_{k}, \\
\sigma_{t}\left(N_{k}\right) & =N_{k}
\end{aligned}
$$

hold for any $k \in \mathbb{Z}^{\nu}$ and $t \in \mathbb{R}$.

Remark 3.2. In fact, the relations (3.5) hold for all $t \in \mathbb{C}$. (See Proposition 2.5 .22 of $[4]$.

Proof of Lemma 3.1. (a) This directly follows from the definitions of $b_{k}^{*}, b_{k}, N_{k}$ and $S_{k}^{z}$ for any $k \in \mathbb{Z}^{\nu}$.

(b) Notice that for $A \in \mathcal{M}_{\Lambda}, \Lambda \in \mathcal{F}$

$$
\begin{aligned}
\delta(A) & :=\left.\frac{d}{d t} \sigma_{t}(A)\right|_{t=0} \\
& =-i\left[H_{\Lambda^{\prime}}, A\right], \Lambda \subset \Lambda^{\prime} .
\end{aligned}
$$

Choosing $A=b_{k}^{*}$ in (3.6), we have

$$
\begin{aligned}
\delta\left(b_{k}^{*}\right) & =\frac{i \beta}{2} \sum_{\substack{l \in \mathbb{Z}^{\nu} \\
|l-k|=1}} S_{l}^{z}\left[S_{k}^{z}, b_{k}^{*}\right] \\
& =i \beta \sum_{\substack{l \in \Xi^{\nu} \\
|l-k|=1}} S_{l}^{z} b_{k}^{*} .
\end{aligned}
$$

Here we have used (3.4) in the second equality. Thus we get

$$
\sigma_{t}\left(b_{k}^{*}\right)=\exp \left(i \beta t \sum_{\substack{l \in Z^{\nu} \\|l-k|=1}} S_{l}^{z}\right) b_{k}^{*} .
$$

The other relations are obtained by the similar calculation.

We define a (diagonal) subalgebra $\mathcal{M}_{d}$ of $\mathcal{M}$, and two Hilbert spaces $\mathcal{H}_{d}$ and $\mathcal{H}_{o d}$ called the diagonal subspace and the off-diagonal subspace, respectively:

$$
\begin{aligned}
& \mathcal{M}_{d}:=\text { the subalgebra generated by }\left\{S_{k}^{z}: k \in \mathbb{Z}^{\nu}\right\}, \\
& \mathcal{H}_{d}:=\text { the closure of } \mathcal{M}_{d} \xi_{0}=\left\{A \xi_{0}: A \in \mathcal{M}_{d}\right\} \text { in } \mathcal{H}, \\
& \mathcal{H}_{o d}:=\text { the orthogonal complement } \mathcal{H}_{d}^{\perp} \text { of } \mathcal{H}_{d} .
\end{aligned}
$$

Remark 3.3. (1) Since $S_{k}^{z}=N_{k}-(1-N)_{k}, k \in \mathbb{Z}^{\nu}$ and $\sigma_{t}\left(N_{k}\right)=N_{k}, \mathcal{M}_{d}$ is the centerizer of $\sigma_{t}$ in the sense that $\sigma_{t}\left(\mathcal{M}_{d}\right)=\mathcal{M}_{d}$ for all $t \in \mathbb{R}$.

(2) Since $\left(S_{k}^{z}\right)^{2}=\mathbf{1}$ for $k \in \mathbb{Z}^{\nu}$, the subspace generated by the vectors of the form

$$
\eta_{\Lambda}:=\left(\prod_{k \in \Lambda} S_{k}^{z}\right) \xi_{0}, \Lambda \in \mathcal{F}
$$


is dense in $\mathcal{H}_{d}$.

(3) $\mathcal{H}=\mathcal{H}_{d} \oplus \mathcal{H}_{o d}$.

(4) $\mathcal{M}_{d} \mathcal{H}_{d} \subset \mathcal{H}_{d}$.

Theorem 3.4 (Action of the generator $H$ on $\mathcal{H}_{d}$ ). For each $k \in \mathbb{Z}^{\nu}$, let $H_{k}$ be defined as in (2.6). Then it acts on $\mathcal{H}_{d}$ as follows: for any $\eta_{\Lambda}=\left(\prod_{l \in \Lambda} S_{l}^{z}\right) \xi_{0} \in$ $\mathcal{H}_{d}, \Lambda \in \mathcal{F}$,

$$
H_{k} \eta_{\Lambda}= \begin{cases}4 \exp \left(-\frac{\beta}{2} \sum_{\substack{l \in-\nu \\|l-k|=1}} S_{k}^{z} S_{l}^{z}\right) \eta_{\Lambda} & \text { if } k \in \Lambda \\ 0 & \text { if } k \notin \Lambda .\end{cases}
$$

Remark 3.5. (1) $T_{t} \mathcal{H}_{d} \subset \mathcal{H}_{d}$ for all $t \in \mathbb{R}$.

(2) For a fixed $k \in \mathbb{Z}^{\nu}$, let $\eta=g\left(S_{k}^{z}, S_{l}^{z} ; l \in \mathbb{Z}^{\nu} \backslash\{k\}\right) \xi_{0}$ and $\eta^{(k)}=$ $g\left(-S_{k}^{z}, S_{l}^{z} ; l \in \mathbb{Z}^{\nu} \backslash\{k\}\right) \xi_{0}$, where $g$ is continuous as a function on $\mathbb{R}^{2}$. Then

$$
\eta-\eta^{(k)}=\left\{\begin{aligned}
2 \eta & \text { if } g \text { is odd for } S_{k}^{z} \\
0 & \text { if } g \text { is even for } S_{k}^{z}
\end{aligned}\right.
$$

The expression (3.7) is rewritten as

$$
H_{k} \eta=2 \exp \left(-\frac{\beta}{2} \sum_{\substack{l \in \in^{\nu} \\|l-k|=1}} S_{k}^{z} S_{l}^{z}\right)\left(\eta-\eta^{(k)}\right) .
$$

This is the Glauber dynamics.

Proof of Theorem 3.4. Let $\eta_{\Lambda}=\left(\prod_{l \in \Lambda} S_{l}^{z}\right) \xi_{0} \in \mathcal{H}_{d}, \Lambda \in \mathcal{F}$. Since for fixed $k \in \Lambda, \prod_{\substack{l \in \Lambda \\ l \neq k}} S_{l}^{z}$ commutes with $H_{k}$ by (3.5) and the definition of $H_{k}$ in (2.6), we consider only

$$
\eta=S_{k}^{z} \xi_{0}=\sigma_{t-i / 4}\left(S_{k}^{z}\right) \xi_{0}
$$

It follows from $\left[N_{k}, S_{k}^{z}\right]=0$ that

$$
H_{k, 3} \eta=0
$$

Next, we consider $H_{k, 1} \eta$. By (3.4) and (3.5), we have

$$
\begin{aligned}
& {\left[\sigma_{t-i / 4}\left(b_{k}^{*}\right)-j\left(\sigma_{t-i / 4}\left(b_{k}\right)\right)\right] \eta } \\
= & \sigma_{t-i / 4}\left(\left[b_{k}^{*}, S_{k}^{z}\right]\right) \xi_{0} \\
= & \sigma_{t-i / 4}\left(-2 b_{k}^{*}\right) \xi_{0} \\
= & -2 \exp \left(\left(i t+\frac{1}{4}\right) \beta \sum_{\substack{l \in \sigma^{-\nu} \\
|l-k|=1}} S_{l}^{z}\right) b_{k}^{*} \xi_{0}
\end{aligned}
$$


and so

$$
\begin{aligned}
& {\left[\sigma_{t+i / 4}\left(b_{k}\right)-j\left(\sigma_{t+i / 4}\left(b_{k}^{*}\right)\right)\right]\left[\sigma_{t-i / 4}\left(b_{k}^{*}\right)-j\left(\sigma_{t-i / 4}\left(b_{k}\right)\right)\right] \eta } \\
= & -2 \exp \left(\left(i t+\frac{1}{4}\right) \beta \sum_{\substack{l \in \mathbb{Z}^{\nu} \\
|l-k|=1}} S_{l}^{z}\right)\left[\sigma_{t+i / 4}\left(b_{k}\right) b_{k}^{*}-b_{k}^{*} j\left(\sigma_{t+i / 4}\left(b_{k}^{*}\right)\right)\right] \xi_{0} \\
= & -2\left[\exp \left(\frac{\beta}{2} \sum_{\substack{l \in \mathbb{Z}^{\nu} \\
|l-k|=1}} S_{l}^{z}\right) b_{k} b_{k}^{*}-\exp \left(-\frac{\beta}{2} \sum_{\substack{l \in \mathbb{Z}^{\nu} \\
|l-k|=1}} S_{l}^{z}\right) b_{k}^{*} b_{k}\right] \xi_{0} \\
(3.9)= & -2 \exp \left(\frac{\beta}{2} \sum_{\substack{l \in \mathbb{Z}^{\nu} \\
|l-k|=1}} S_{l}^{z}\right)(1-N)_{k} \xi_{0}+2 \exp \left(-\frac{\beta}{2} \sum_{\substack{l \in \mathbb{Z}^{\nu} \\
|l-k|=1}} S_{l}^{z}\right) N_{k} \xi_{0} .
\end{aligned}
$$

Here we have used $j\left(\sigma_{t+i / 4}\left(b_{k}^{*}\right)\right) \xi_{0}=\sigma_{t-3 i / 4}\left(b_{k}\right) \xi_{0}$ and $(3.5)$ in the second equality and (3.2) in the third equality.

Notice that by (3.1) and (3.2)

$$
\begin{aligned}
& \exp \left(\frac{\beta}{2} \sum_{\substack{l \in \mathbb{Z}^{\nu} \\
|l-k|=1}} S_{l}^{z}\right)(\mathbf{1}-N)_{k}=\exp \left(-\frac{\beta}{2} \sum_{\substack{l \in \mathbb{Z}^{\nu} \\
|l-k|=1}} S_{k}^{z} S_{l}^{z}\right)(\mathbf{1}-N)_{k}, \\
& \exp \left(-\frac{\beta}{2} \sum_{\substack{l \in \mathbb{Z}^{\nu} \\
|l-k|=1}} S_{l}^{z}\right) N_{k}=\exp \left(-\frac{\beta}{2} \sum_{\substack{l \in Z^{\nu} \nu \\
|l-k|=1}} S_{k}^{z} S_{l}^{z}\right) N_{k} .
\end{aligned}
$$

Substituting (3.10) into (3.9), we get

$$
\begin{aligned}
& {\left[\sigma_{t+i / 4}\left(b_{k}\right)-j\left(\sigma_{t+i / 4}\left(b_{k}^{*}\right)\right)\right]\left[\sigma_{t-i / 4}\left(b_{k}^{*}\right)-j\left(\sigma_{t-i / 4}\left(b_{k}\right)\right)\right] \eta } \\
= & -2 \exp \left(-\frac{\beta}{2} \sum_{\substack{l \in \mathbb{Z}^{\nu} \\
|l-k|=1}} S_{k}^{z} S_{l}^{z}\right)(1-N)_{k} \xi_{0}+2 \exp \left(-\frac{\beta}{2} \sum_{\substack{l \in \pi^{\nu} \\
|l-k|=1}} S_{k}^{z} S_{l}^{z}\right) N_{k} \xi_{0} \\
= & 2 \exp \left(-\frac{\beta}{2} \sum_{\substack{l \in \mathbb{Z}^{\nu} \\
|l-k|=1}} S_{k}^{z} S_{l}^{z}\right) S_{k}^{z} \xi_{0} \\
= & 2 \exp \left(-\frac{\beta}{2} \sum_{\substack{l \in \mathbb{Z}^{\nu} \\
|l-k|=1}} S_{k}^{z} S_{l}^{z}\right) \eta .
\end{aligned}
$$

Thus for $\eta_{\Lambda}=\left(\prod_{l \in \Lambda} S_{l}^{z}\right) \xi_{0}$, since $\int f(t) d t=1$, we have

$$
H_{k, 1} \eta_{\Lambda}= \begin{cases}2 \exp \left(-\frac{\beta}{2} \sum_{\substack{l \in \in \mathbb{Z}^{\nu} \\|l-k|=1}} S_{k}^{z} S_{l}^{z}\right) \eta_{\Lambda} & \text { if } k \in \Lambda \\ 0 & \text { if } k \notin \Lambda .\end{cases}
$$

By the similar calculation we can also check

$$
H_{k, 2} \eta_{\Lambda}=H_{k, 1} \eta_{\Lambda}
$$

The relation (3.7) follows from (3.8), (3.11) and (3.12). The proof is completed. 
In the rest of this paper, $b_{k}^{\sharp}$ is either $b_{k}^{*}$ or $b_{k}$. Recall that

$$
\left(S_{k}^{z}\right)^{2}=\mathbf{1}, b_{k}^{2}=\left(b_{k}^{*}\right)^{2}=0, b_{k}^{*} b_{k}=N_{k}=\frac{1}{2}\left(1+S_{k}^{z}\right), \text { etc. }
$$

Let $A \in \mathcal{M}$ be a monomial in the algebra generated by $\left\{b_{k}, b_{k}^{*}, S_{k}^{z}: k \in \mathbb{Z}^{\nu}\right\}$. Then $A \xi_{0}$ can be written as a linear combination of the vectors of the form

$$
\eta_{\Lambda_{1}, \Lambda_{2}}:=\left(\prod_{l \in \Lambda_{2}} b_{l}^{\sharp}\right)\left(\prod_{m \in \Lambda_{1}} S_{m}^{z}\right) \xi_{0},
$$

where $\Lambda_{1}, \Lambda_{2} \in \mathcal{F}$ are disjoint. Clearly the family of the above vectors is dense in $\mathcal{H}$. Recall that the diagonal subspace $\mathcal{H}_{d}$ is spanned by the vectors of the form $\eta_{\Lambda, \emptyset}:=\eta_{\Lambda}=\left(\prod_{l \in \Lambda} S_{l}^{z}\right) \xi_{0}, \Lambda \in \mathcal{F}$.

Lemma 3.6. (a) The Hilbert space $\mathcal{H}_{\text {od }}$ is the closure of the subspace spanned by the vectors of the form

$$
\eta_{\Lambda_{1}, \Lambda_{2}}=\left(\prod_{l \in \Lambda_{2}} b_{l}^{\sharp}\right)\left(\prod_{m \in \Lambda_{1}} S_{m}^{z}\right) \xi_{0},
$$

where $\Lambda_{1}, \Lambda_{2} \in \mathcal{F}$ are disjoint and $\Lambda_{2} \neq \emptyset$.

(b) $\mathcal{M}_{d} \mathcal{H}_{o d} \subset \mathcal{H}_{\text {od }}$.

Recall $\mathcal{M}_{d} \mathcal{H}_{d} \subset \mathcal{H}_{d}$ (Remark $\left.3.3(4)\right)$.

Proof of Lemma 3.6. (a) Let $\eta_{\Lambda_{1}, \Lambda_{2}}=\left(\prod_{l \in \Lambda_{2}} b_{l}^{\sharp}\right)\left(\prod_{m \in \Lambda_{1}} S_{m}^{z}\right) \xi_{0}$ for $\Lambda_{1}, \Lambda_{2} \in \mathcal{F}$, $\Lambda_{1} \cap \Lambda_{2}=\emptyset$ and $\Lambda_{2} \neq \emptyset$. Notice that for any $\Lambda_{3} \in \mathcal{F}$

$$
\left\langle\eta_{\Lambda_{3}}, \eta_{\Lambda_{1}, \Lambda_{2}}\right\rangle=\left\langle\xi_{0},\left(\prod_{l \in \Lambda_{2}} b_{l}^{\sharp}\right)\left(\prod_{m \in \Lambda_{1} \Delta \Lambda_{3}} S_{m}^{z}\right) \xi_{0}\right\rangle,
$$

where we have used the KMS condition and $\left(S_{k}^{z}\right)^{2}=\mathbf{1}$.

Since $\mathcal{H}_{d}$ is spanned by the vectors of the form $\left(\prod_{l \in \Lambda} S_{l}^{z}\right) \xi_{0}, \Lambda \in \mathcal{F}$, it suffices to show that

$$
\left\langle\xi_{0},\left(\prod_{l \in \Lambda_{2}} b_{l}^{\sharp}\right)\left(\prod_{m \in \Lambda_{1} \triangle \Lambda_{3}} S_{m}^{z}\right) \xi_{0}\right\rangle=0 \text { for } \Lambda_{2} \neq \emptyset .
$$

Using the KMS condition, we have

$$
\left\langle\xi_{0}, b_{l}^{\sharp} S_{l}^{z} \eta_{\Lambda_{4}, \Lambda_{5}}\right\rangle=\left\langle\xi_{0}, S_{l}^{z} b_{l}^{\sharp} \eta_{\Lambda_{4}, \Lambda_{5}}\right\rangle
$$

for $l \notin \Lambda_{4} \cup \Lambda_{5}, \Lambda_{4} \cap \Lambda_{5}=\emptyset, \Lambda_{4}, \Lambda_{5} \in \mathcal{F}$, and so

$$
\left\langle\xi_{0},\left[b_{l}^{\sharp}, S_{l}^{z}\right] \eta_{\Lambda_{4}, \Lambda_{5}}\right\rangle=0,
$$

which implies (3.13). We obtain from (3.4) that

$$
\left\langle\xi_{0}, b_{l}^{\sharp} \eta_{\Lambda_{4}, \Lambda_{5}}\right\rangle=0 \text { if } l \notin \Lambda_{4} \cup \Lambda_{5} .
$$

The part ( $a$ ) of lemma is proved.

(b) This follows from the facts $S_{l}^{z} b_{l}^{*}=b_{l}^{*}, S_{l}^{z} b_{l}=-b_{l}$. 
Theorem 3.7 (Action of the generator $H$ on $\mathcal{H}_{o d}$ ). For each $k \in \mathbb{Z}^{\nu}$, let $H_{k}$ be defined as in (2.6). Then it acts on $\mathcal{H}_{\text {od }}$ as follows: for any

$$
\eta_{\Lambda_{1}, \Lambda_{2}}=\left(\prod_{l \in \Lambda_{2}} b_{l}^{\sharp}\right)\left(\prod_{m \in \Lambda_{1}} S_{m}^{z}\right) \xi_{0},
$$

$\Lambda_{1}, \Lambda_{2} \in \mathcal{F}, \Lambda_{1} \cap \Lambda_{2}=\emptyset$ and $\Lambda_{2} \neq \emptyset$,

$$
H_{k} \eta_{\Lambda_{1}, \Lambda_{2}}=\left\{\begin{array}{cl}
0 & \text { if } k \notin \Lambda_{1} \cup \Lambda_{2}, \\
{\left[\mathbf{1}+2 \cosh \left(\frac{\beta}{2} \sum_{\substack{l \in Z^{\nu} \\
|l-k|=1}} \varepsilon_{l} S_{k}^{z} S_{l}^{z}\right)\right] \eta_{\Lambda_{1}, \Lambda_{2}}} & \text { if } k \in \Lambda_{2}, \\
4 \exp \left(-\frac{\beta}{2} \sum_{\substack{l \in \mathbb{Z}^{2} \nu \\
|l-k|=1}} \varepsilon_{l} S_{k}^{z} S_{l}^{z}\right) \eta_{\Lambda_{1}, \Lambda_{2}} & \text { if } k \in \Lambda_{1} .
\end{array}\right.
$$

Here $\varepsilon_{l}=-1$ if $l \in \Lambda_{2}$ and $\varepsilon_{l}=1$ if $l \notin \Lambda_{2}$.

Proof. Let

$$
\eta_{\Lambda_{1}, \Lambda_{2}}=\left(\prod_{l \in \Lambda_{2}} b_{l}^{\sharp}\right)\left(\prod_{m \in \Lambda_{1}} S_{m}^{z}\right) \xi_{0}
$$

for $\Lambda_{1}, \Lambda_{2} \in \mathcal{F}, \Lambda_{1} \cap \Lambda_{2}=\emptyset$ and $\Lambda_{2} \neq \emptyset$. Recall that $\prod_{\substack{l \in \Lambda_{1} \\ l \neq k}} S_{l}^{z}$ and $\prod_{\substack{l \in \Lambda_{2} \\ l \neq k}} b_{l}^{\sharp}$ commute with $H_{k}$. If $k \notin \Lambda_{1} \cup \Lambda_{2}$, then

$$
H_{k} \eta_{\Lambda_{1}, \Lambda_{2}}=0 \text {. }
$$

To prove the case $k \in \Lambda_{2}$, let

$$
\eta_{\Lambda_{1}, \Lambda_{2}}=b_{k}^{*}\left(\prod_{\substack{l \in \Lambda_{2} \\ l \neq k}} b_{l}^{\sharp}\right)\left(\prod_{m \in \Lambda_{1}} S_{m}^{z}\right) \xi_{0} .
$$

Since $\left[N_{k}, b_{k}^{*}\right]=b_{k}^{*}$ and $\int f(t) d t=1$, it is easily checked that

$$
H_{k, 3} \eta_{\Lambda_{1}, \Lambda_{2}}=\eta_{\Lambda_{1}, \Lambda_{2}}
$$

Also we get from the identity $\left(b_{k}^{*}\right)^{2}=0$ that

$$
H_{k, 1} \eta_{\Lambda_{1}, \Lambda_{2}}=0 \text {. }
$$

Now, we calculate $H_{k, 2} \eta_{\Lambda_{1}, \Lambda_{2}}$. We will again adopt the similar calculations used to get the relation (3.11) in the proof of Theorem 3.4. By (3.5) and $\left[b_{k}, b_{k}^{*}\right]=-S_{k}^{z}$, we have

$$
\begin{aligned}
& {\left[\sigma_{t-i / 4}\left(b_{k}\right)-j\left(\sigma_{t-i / 4}\left(b_{k}^{*}\right)\right)\right] b_{k}^{*} \xi_{0} } \\
= & \exp \left(-\left(i t+\frac{1}{4}\right) \beta \sum_{\substack{l \in Z^{\nu} \\
|l-k|=1}} S_{l}^{z}\right)\left[b_{k}, b_{k}^{*}\right] \xi_{0} \\
= & -\exp \left(-\left(i t+\frac{1}{4}\right) \beta \sum_{\substack{l \in Z^{\nu} \\
|l-k|=1}} S_{l}^{z}\right) S_{k}^{z} \xi_{0}
\end{aligned}
$$


and hence

$$
\begin{aligned}
& {\left[\sigma_{t+i / 4}\left(b_{k}^{*}\right)-j\left(\sigma_{t+i / 4}\left(b_{k}\right)\right)\right]\left[\sigma_{t-i / 4}\left(b_{k}\right)-j\left(\sigma_{t-i / 4}\left(b_{k}^{*}\right)\right)\right] b_{k}^{*} \xi_{0}} \\
& =-\exp \left(-\left(i t+\frac{1}{4}\right) \beta \sum_{\substack{l \in=\nu \\
|l-k|=1}} S_{l}^{z}\right)\left[\sigma_{t+i / 4}\left(b_{k}^{*}\right) S_{k}^{z}-S_{k}^{z} j\left(\sigma_{t+i / 4}\left(b_{k}\right)\right)\right] \xi_{0} \\
& =\left[-\exp \left(-\frac{\beta}{2} \sum_{\substack{l \in Z^{\nu} \\
|l-k|=1}} S_{l}^{z}\right) b_{k}^{*} S_{k}^{z}+\exp \left(\frac{\beta}{2} \sum_{\substack{l \in \nu \nu \\
|l-k|=1}} S_{l}^{z}\right) S_{k}^{z} b_{k}^{*}\right] \xi_{0} \\
& =\exp \left(-\frac{\beta}{2} \sum_{\substack{l \in \in^{\prime} \\
|l-k|=1}} S_{k}^{z} S_{l}^{z}\right) b_{k}^{*} \xi_{0}+\exp \left(\frac{\beta}{2} \sum_{\substack{l \in=\nu \\
|l-k|=1}} S_{k}^{z} S_{l}^{z}\right) b_{k}^{*} \xi_{0} \\
& =\left[\exp \left(-\frac{\beta}{2} \sum_{\substack{l \in J^{\nu} \\
|l-k|=1}} S_{k}^{z} S_{l}^{z}\right)+\exp \left(\frac{\beta}{2} \sum_{\substack{l \in J^{\nu} \\
|l-k|=1}} S_{k}^{z} S_{l}^{z}\right)\right] b_{k}^{*} \xi_{0}
\end{aligned}
$$

where we used (3.3) in the third equality.

Applying $b_{k}^{\sharp} \exp \left( \pm \frac{\beta}{2} S_{k}^{z}\right)=\exp \left(\mp \frac{\beta}{2} S_{k}^{z}\right) b_{k}^{\sharp}$ to the above, and since the function $f(t)$ is normalized admissible, we have

$$
\begin{aligned}
& H_{k, 2} \eta_{\Lambda_{1}, \Lambda_{2}}=\left[\exp \left(-\frac{\beta}{2} \sum_{\substack{l \in \in^{\nu} \\
|l-k|=1}} \varepsilon_{l} S_{k}^{z} S_{l}^{z}\right)+\exp \left(\frac{\beta}{2} \sum_{\substack{l \in \in^{z} \\
|l-k|=1}} \varepsilon_{l} S_{k}^{z} S_{l}^{z}\right)\right] \eta_{\Lambda_{1}, \Lambda_{2}} \\
& =2 \cosh \left(\frac{\beta}{2} \sum_{\substack{l \in \sim^{\nu} \\
|l-k|=1}} \varepsilon_{l} S_{k}^{z} S_{l}^{z}\right) \eta_{\Lambda_{1}, \Lambda_{2}},
\end{aligned}
$$

where $\varepsilon_{l}=-1$ if $l \in \Lambda_{2}$ and $\varepsilon_{l}=1$ if $l \notin \Lambda_{2}$.

For $\eta_{\Lambda_{1}, \Lambda_{2}}=b_{k}\left(\prod_{\substack{l \in \Lambda_{2} \\ l \neq k}} b_{l}^{\sharp}\right)\left(\prod_{m \in \Lambda_{1}} S_{m}^{z}\right) \xi_{0}$, the similar calculation as the above gives

$$
\begin{aligned}
& H_{k, 3} \eta_{\Lambda_{1}, \Lambda_{2}}=\eta_{\Lambda_{1}, \Lambda_{2}} \\
& H_{k, 1} \eta_{\Lambda_{1}, \Lambda_{2}}=0 \\
& H_{k, 2} \eta_{\Lambda_{1}, \Lambda_{2}}=2 \cosh \left(\frac{\beta}{2} \sum_{\substack{l \in=\nu \\
|l-k|=1}} \varepsilon_{l} S_{k}^{z} S_{l}^{z}\right) \eta_{\Lambda_{1}, \Lambda_{2}}
\end{aligned}
$$

Hence we get

$$
H_{k} \eta_{\Lambda_{1}, \Lambda_{2}}=\left[\mathbf{1}+2 \cosh \left(\frac{\beta}{2} \sum_{\substack{l \in \sigma^{\nu} \\|l-k|=1}} \varepsilon_{l} S_{k}^{z} S_{l}^{z}\right)\right] \eta_{\Lambda_{1}, \Lambda_{2}}
$$

Next, to prove the case $k \in \Lambda_{1}$, let

$$
\eta_{\Lambda_{1}, \Lambda_{2}}=S_{k}^{z}\left(\prod_{l \in \Lambda_{2}} b_{l}^{\sharp}\right)\left(\prod_{\substack{m \in \Lambda_{1} \\ m \neq k}} S_{m}^{z}\right) \xi_{0} .
$$


By the similar calculation used to (3.16), (3.17) and (3.18), we have

$$
\begin{aligned}
H_{k, 3} \eta_{\Lambda_{1}, \Lambda_{2}} & =0, \\
H_{k, 1} \eta_{\Lambda_{1}, \Lambda_{2}} & =H_{k, 2} \eta_{\Lambda_{1}, \Lambda_{2}} \\
& =2\left(\prod_{l \in \Lambda_{2}} b_{l}^{\sharp}\right)\left(\prod_{\substack{m \in \Lambda_{1} \\
m \neq k}} S_{m}^{z}\right) \exp \left(-\frac{\beta}{2} \sum_{\substack{l \in Z^{\nu} \\
|i-k|=1}} \varepsilon_{l} S_{k}^{z} S_{l}^{z}\right) S_{k}^{z} \xi_{0},
\end{aligned}
$$

where $\varepsilon_{l}$ is defined as in (3.18). Applying (3.3) to (3.21), we get

$$
\begin{aligned}
H_{k, 1} \eta_{\Lambda_{1}, \Lambda_{2}} & =H_{k, 2} \eta_{\Lambda_{1}, \Lambda_{2}} \\
& =2 \exp \left(-\frac{\beta}{2} \sum_{\substack{l \in Z^{\nu} \\
|l-k|=1}} \varepsilon_{l} S_{k}^{z} S_{l}^{z}\right) \eta_{\Lambda_{1}, \Lambda_{2}} .
\end{aligned}
$$

Thus

$$
H_{k} \eta_{\Lambda_{1}, \Lambda_{2}}=4 \exp \left(-\frac{\beta}{2} \sum_{\substack{l \in Z^{\nu} \\|l-k|=1}} \varepsilon_{l} S_{k}^{z} S_{l}^{z}\right) \eta_{\Lambda_{1}, \Lambda_{2}} .
$$

The proof is completed.

Remark 3.8. (1) $T_{t} \mathcal{H}_{o d} \subset \mathcal{H}_{o d}$ for all $t \in \mathbb{R}$.

(2) For any $\eta \in \mathcal{H}_{o d},\langle\eta, H \eta\rangle \geq\|\eta\|^{2}$. In particular, for any $\eta \in \mathcal{H}_{o d}, T_{t} \eta$ decays to zero exponentially fast as $t \rightarrow \infty$.

\section{References}

[1] L. Accardi and S. Koyzyrev, Lectures on quantum interacting particle systems, Quantum interacting particle systems (Trento, 2000), 1-195, QP-PQ: Quantum Probab. White Noise Anal., 14, World Sci. Publ., River Edge, NJ, 2002.

[2] S. Albeverio and R. Høegh-Krohn, Dirichlet forms and Markovian semigroups on $C^{*}$ algebras, Comm. Math. Phys. 56 (1997), 173-187.

[3] C. Bahn, C. K. Ko, and Y. M. Park, Dirichlet forms and symmetric Markovian semigroups on CCR algebras with respect to quasi-free states, J. Math. Phys. 44 (2003), no. 2, 723-753.

[4] O. Bratteli and D. W. Robinson, Operator Algebras and Quantum Statistical Mechanics. 2, Equilibrium states. Models in quantum statistical mechanics. Second edition. Texts and Monographs in Physics. Springer-Verlag, Berlin, 1997.

[5] R. Carbone, F. Fagnola, and S. Hachicha, Generic quantum Markov semigroups: the Gaussian quage invariant case, preprint.

[6] F. Cipriani, Dirichlet forms and Markovian semigroups on standard forms of von Neumann algebras, J. Funct. Anal. 147 (1997), no. 2, 259-300.

[7] D. Goderis and C. Maes, Constructing quantum dissipations and their reversible states from classical interacting spin systems, Ann. Inst. H. Poincare Phys. Theor. 55 (1991), no. 3, 805-828.

[8] Y. M. Park, Construction of Dirichlet forms on standard forms of von Neumann algebras, Infin. Dimens. Anal. Quantum Probab. Relat. Top. 3 (2000), no. 1, 1-14.

[9] K. R. Parthasarathy, An Introduction to Quantum Stochastic Calculus, Birkhäuser, Basel, 1992. 


\author{
VENI CHOI \\ Division of General Studies \\ AJOU UNIVERSITY \\ SUWON 443-749, KorEA \\ E-mail address: veni@ajou.ac.kr \\ Chul KI Ko \\ UNIVERSITY COLLEGE \\ YONSEI UNIVERSITY \\ SeOul 120-749, Korea \\ E-mail address: kochulki@yonsei.ac.kr \\ YONG MOON PARK \\ Department of Mathematics \\ YONSEI UNIVERSITY \\ SEOUL 120-749, KOREA \\ E-mail address: ympark@yonsei.ac.kr
}

